\title{
Added value of thin axial MR cuts in the diagnosis of several meniscal tears: how far are they reliable?
}

\author{
Yasser I. Abd Elkhalek ${ }^{1 *}$, Moustafa Abdel Kawi ${ }^{2}$ and Merhan Nasr ${ }^{3}$
}

\begin{abstract}
Background: The purpose of the study is to assess the added value of thin-cut (1 $\mathrm{mm}$ ) axial magnetic resonance (MR) fat-saturated proton density (FS PD) weighted images (WIs) in the diagnosis of different types of meniscal tears. The study was conducted between January 2016 and August 2017 at a major private center group. This study included 28 patients with clinical suspicion of meniscal tear of age between 30 and 48 years including 19 males and 9 females. Axial 1-mm FS PD WIs were performed in addition to the conventional MR sequences. The type and classification of the meniscal tear were then diagnosed by a group of highly experienced musculoskeletal radiologists. The group assessed the morphology and classification of tears. The diagnosis was then confirmed by arthroscopy as a gold-standard reference.
\end{abstract}

Results: Meniscal tears were found in 23 out of 28 patients. Twenty-seven meniscal tears were found in the 23 patients: 2 root attachment radial tears (6.9\%), 8 radial tears (27.6\%), 7 longitudinal [vertical] tears (24\%), 3 horizontal tears (10\%), 5 displaced bucket handle tears (17\%), 1 case of flab tear (3.4\%), 1 case of meniscal fraying (3.4\%), and 2 lesions which were diagnosed as artifacts and confirmed negative for tears by arthroscopy (6.8\%). Diagnostic performance was as follows: sensitivity $96.3 \%$, positive predictive value $100 \%$, accuracy $96.6 \%$, prevalence $93 \%$, specificity $100 \%$, and negative predictive value $66.67 \%$.

Conclusion: The study declared high diagnostic performance of thin-cut fat-saturated PD WIs in the diagnosis of different types of meniscal tears regarding their classifications and morphology which is crucial for further management.

Keywords: Magnetic resonance imaging, Thin axial, meniscal tears

\section{Background}

The knee menisci serve as an axial load distributor and shock absorber and help in lubrication and nutrient distribution in the joint [1].

Meniscal tears are one of the most common indications for MRI of the knee and one of the commonest causes of knee pain. Meniscal tears can occur secondary to trauma or degeneration. Twisting-type or fast rotatory trauma is the commonest cause of injury, and this for sure occurs in young individuals while the degenerative type occurs in adults [1].

MRI is the best radiological method for diagnosis of different types of tears, and it is far more accurate than

\footnotetext{
* Correspondence: yasserib77@gmail.com

${ }^{1}$ Radiodiagnosis Department, Ain Shams University, 3/4 Borg Elhamd Anwer

Elmofty Street, Nasr City, Cairo, Egypt

Full list of author information is available at the end of the article
}

other modalities like sonography or computed tomography. The characteristic C-shaped fibrocartilaginous semilunar disc appearance of the meniscus makes the accurate diagnosis of meniscal tear a challenge. Also, the presence of normal variants like discoid meniscus, meniscal ossicle, and meniscal flounce, as well as chondrocalcinosis, makes the diagnosis more difficult and needs much attention. The type and extensions of tear are crucial in the diagnosis before arthroscopy to determine the best repair plan [2]. A root attachment tear, for example, is a radial tear occurring at the meniscal root. This type of tear is difficult to diagnose in MRI and needs special attention to the root [3].

The shape and orientation of the meniscal tears depend on direct and indirect signs. The proton density fat-suppressed sequences in coronal and sagittal views are the widely used sequences in the tear diagnosis. The 
sagittal view is used to detect signs of meniscal tears like a high signal longitudinally oriented line in longitudinal tears or a horizontally oriented line in horizontal tears. Those tears are easier to diagnose than the radial tear that depends on more indirect signs like the truncated triangle sign, cleft sign, and marching cleft sign, as well as the ghost meniscal sign. In bucket handle tear also, there are indirect signs like loss of normal bow-tie appearance of the meniscal body in the peripheral sagittal planes and double posterior cruciate ligament (PCL) sign in the midsagittal images. Other indirect signs include different patterns of bone marrow edema, a parameniscal cyst in horizontal tear and meniscal extrusion [4].

The indirect signs are not specific for tears. A double PCL sign can be seen when there is hypertrophy of the ligament of Humphry. Sometimes, the hypertrophied anterior inter-meniscal ligament simulates vertical cleavage tear of the anterior horn. The lateral meniscal tears are harder to diagnose as the meniscus is smaller and having a complex attachment with the joint, and so, some of the normal anatomic structures can be mistaken as a tear [5].

Traditionally, sagittal and coronal MRI sequences are used due to the orientation of the meniscus in a parallel plane to the joint articular surface and so axial scans presumed to be not sensitive in the diagnosis of the meniscal tear. We believe this is not true now because the advancements in high-field MRI, application of better fat-suppressed images, very thin-cut images, and lower field of view improve the detection of tears in the axial images. Thin-cut fat-suppressed images can accurately diagnose the type of the tear, and moreover, it can directly demonstrate its extent and actual morphology unlike the other plans [6].

\section{Materials and methods}

The study was conducted between January 2016 and August 2017 at a private center group. We used the MR General Electric ${ }^{\circ}$, Signa system (Milwaukee, WI, USA) operating at $1.5 \mathrm{~T}$, using a knee coil. The patients were lying supine in all cases with an extended knee. The axial sequence used was FSE PD weighted image (WI) with fat suppression $(\mathrm{TE}=20-30 \mathrm{~ms} ; \mathrm{TR}=3000 \mathrm{~ms}$; thickness $=1 \mathrm{~mm}$; inter-slice gap $=0.15 \mathrm{~mm} ; \mathrm{FA}=180^{\circ}$; FOV $=180 \times 180$; matrix, $288 \times 224$; imaging time $=6$ $\mathrm{min}$ ). We used a sagittal [or coronal] localizer while ensuring the parallel plane to the meniscus in the taken localizer (Fig. 1).

This study included 28 patients with clinical suspicion of meniscal tear aged between 30 and 48 years (mean age 35 years) including 19 men (68\%) and 9 women (32\%). Axial 1-mm FS PD WIs were performed in addition to the conventional MR sequences. The type and classification of the meniscal tear were then diagnosed by a group of experienced musculoskeletal radiologists. They assessed the morphology and classification of tears. The meniscal tear diagnostic criteria were abnormal morphology of the meniscus or abnormal signal intensity within the meniscal substance. The diagnosis was then confirmed by arthroscopy as a gold-standard reference.

Cases that underwent previous knee surgical intervention or arthroscopy were excluded, and we excluded cases aging more than 45 years. No patients had contraindications for MRI.

We used SPSS V. 13 in the statistical analysis. The mean and standard deviation were presented for continuous variables. Frequency and percentage were expressed for categorical data. We calculated the diagnostic performance of fat-suppressed thin-cut PD WIs used in the detection of different types of meniscal tears compared to the arthroscopy results. The significant statistical difference was at a $p$ value less than 0.05 .

\section{Results}

Meniscal tears were found in 23 out of 28 patients. Twenty-seven meniscal tears were found in the 23 patients as follows:

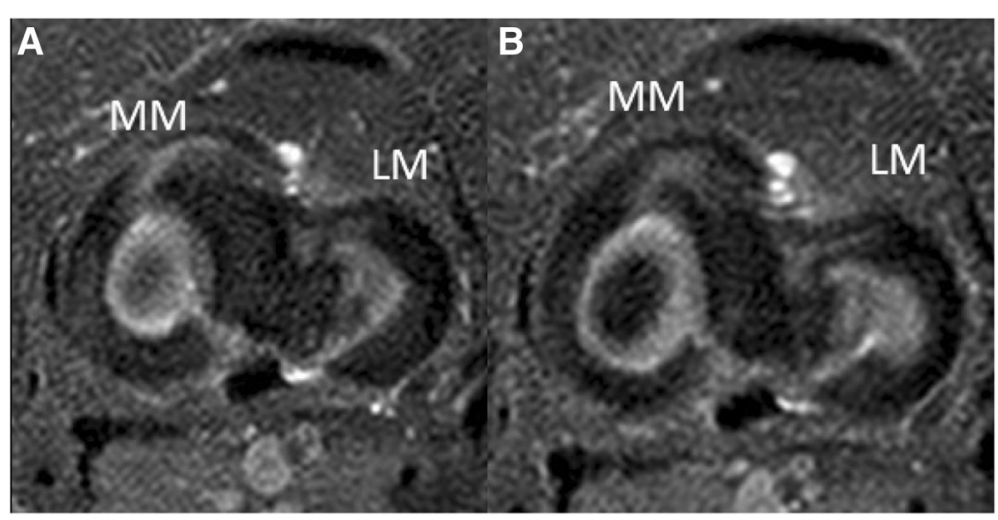

Fig. 1 a, b Two consecutive thin $(0.5 \mathrm{~mm})$ axial PD fat-saturated images revealed normal meniscal configuration. $M M=$ medial meniscus and $L M=$ lateral meniscus 
- 2 root attachment medial meniscal tears $(6.9 \%)$ (Fig. 2).

- 8 radial tears $(27.6 \%)$ :

- 2 complete radial tears (Fig. 3)

- 6 incomplete radial tears: 4 true radial (Fig. 4) and 2 oblique radial [parrot peak] (Fig. 5)

- 7 longitudinal vertical tears (24\%) (Fig. 6)

- 3 horizontal tears (10\%).

- 5 displaced bucket handle tears (17\%) (4 medial meniscal and 1 lateral meniscal) (Figs. 7 and 8)

- 1 case of flap tear (3.4\%) (Fig. 9)

- 1 case of meniscal fraying (3.4\%)

- 2 lesions were diagnosed as artifacts and were confirmed negative for tears by arthroscopy (6.8\%)

The medial meniscus was involved in 19 tears (70\%) and lateral meniscus in 8 tears (30\%). One horizontal tear was missed and found in arthroscopy. A case had medial meniscal fraying and radial tear. One case shows a flap tear in the medial meniscus and another small radial root tear in the medial meniscus. Another case has 2 lesions: one bucket handle tear in the lateral meniscus and a radial tear in the medial meniscus. The mean age was 34 years for longitudinal tear, 40 years for horizontal tear, 34 years for radial tear, and 34 years for bucket handle tear. Diagnostic performance was as follows: sensitivity $96.3 \%$, PPV $100 \%$, accuracy $96.6 \%$, prevalence $93 \%$, specificity $100 \%$, and NPV $66.67 \%$.

\section{Discussion}

The diagnosis of meniscal lesions is a classic indication for knee MRI since the MRI invention. Traditionally, the axial, sagittal, and coronal images were used in the diagnosis. Because in old systems and technology used in the past, the limited number of sequences was used in the diagnosis and with thick cuts $(4-5 \mathrm{~mm})$. Now, high-field MRI and the advances in MRI technology had lessened

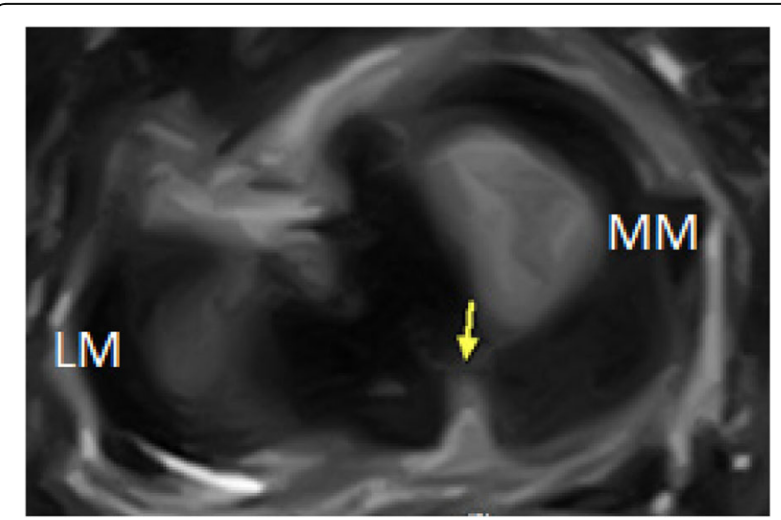

Fig. 2 Thin axial fat-saturated PD WI revealed a posterior horn medial meniscus root attachment tear (yellow arrow). $\mathrm{MM}=$ medial meniscus and $\mathrm{LM}=$ lateral meniscus

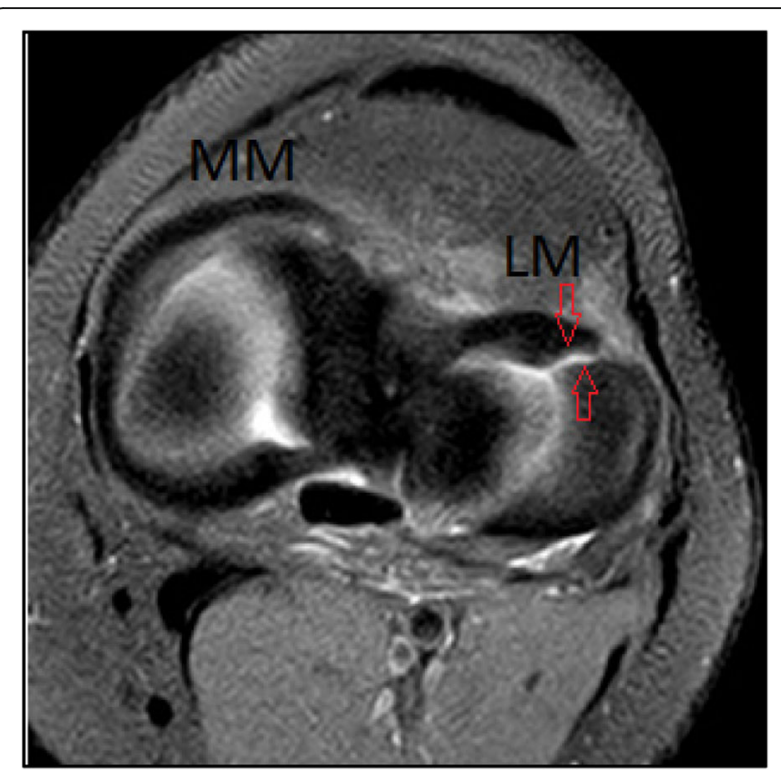

Fig. 3 Axial PD fat-saturated images revealed a complete radial tear of the lateral meniscus (at the junction between the anterior horn and body, red arrows), extending from the free meniscal edge to the capsular surface. $\mathrm{LM}=$ lateral meniscus and MM = medial meniscus

the time used in each sequence and make much better resolution even with very thin cuts. Due to old MRI system limitations and meniscal orientation in axial plane parallel to the femoral and tibial articular surfaces, axial cuts were not an interest in meniscal tear diagnosis, unlike the sagittal and coronal planes. The sagittal and coronal planes can diagnose accurately most of the meniscal lesions, yet, in some cases, it can misdiagnose tears because it uses some indirect signs in diagnosis that could be non-specific in some cases. Moreover, the

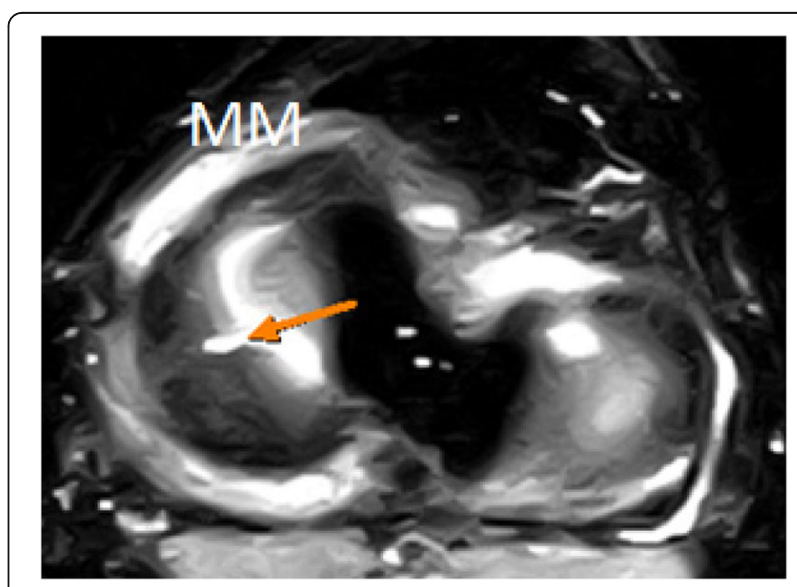

Fig. 4 Thin axial fat-saturated PD WI revealed a small incomplete radial tear related to the body of the medial meniscus (orange arrow) involving the free meniscal edge (sparing the meniscocapsular attachment). MM = medial meniscus 


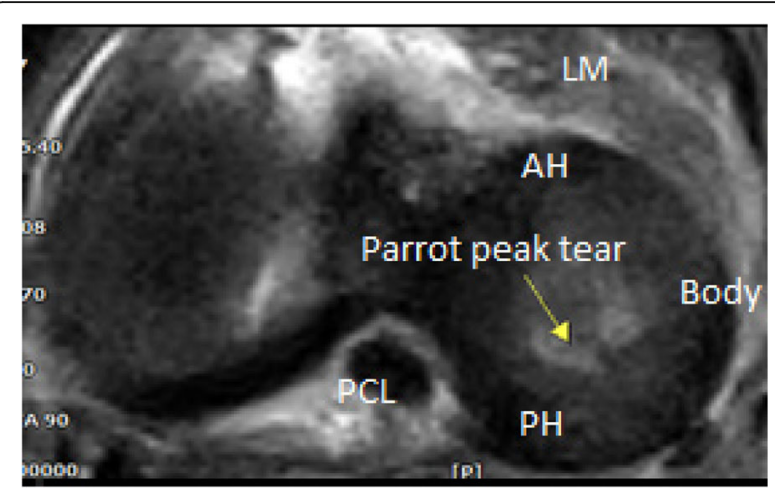

Fig. 5 Axial fat-saturated PD WI revealed small parrot peak tear (arrow) related to the free meniscal edge at the junction between the body and posterior horn. $\mathrm{LM}=$ lateral meniscus, $\mathrm{AH}=$ anterior horn, $\mathrm{PH}=$ posterior horn, and $\mathrm{PCL}=$ posterior cruciate ligament

exact morphology and extent of the lesion are not readily seen in those planes and that raises the importance of the axial thin-cut fat-suppressed PD sequence in the diagnosis as it can overcome these limitations and has an added value in accurate diagnosis. The morphology of the tear is important not only in the management of the presence or absence of tear because the decision of suturing or resection of the affected horn depends on its morphology [7].

MRI sensitivity in the diagnosis of medial meniscal tears was found to be about $87-97 \%$ and specificity about $87-98 \%$ while for lateral meniscal tears sensitivity was $72-93 \%$ and specificity $89-99 \%$ according to previous studies [7]. This range of difference in the results is due to the different types of sequences used in the diagnosis and the sample size in previous studies, as well as interobserver variability [8].

Araki et al. made a comparative study between 3D gradient-recalled acquisition in the steady state (GRASS)

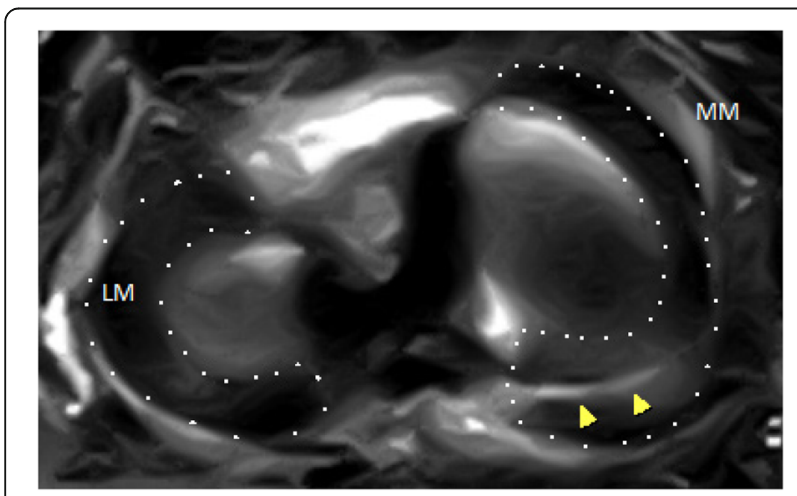

Fig. 6 Thin axial fat-saturated PD WI revealed a longitudinal nondisplaced vertical tear related to the posterior horn of the medial meniscus (arrow heads). $\mathrm{MM}=$ medial meniscus and $\mathrm{LM}=$ lateral meniscus

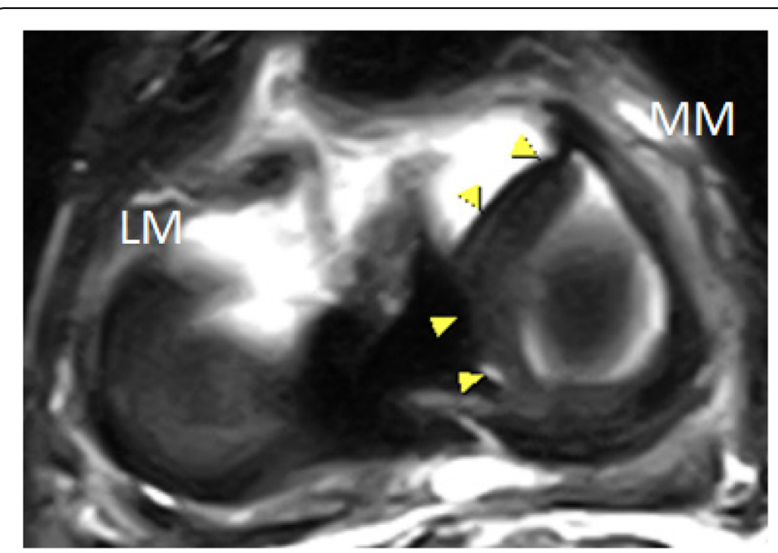

Fig. 7 Thin axial fat-saturated PD WI revealed a displaced medial meniscal bucket handle tear. Note the centrally displaced torn inner meniscal fragment (arrow heads). MM = medial meniscus and $\mathrm{LM}=$ lateral meniscus

0.7 -mm thickness versus sagittal and coronal images and found that it had $97 \%$ sensitivity and $96 \%$ specificity [9].

Tarhan et al. also found that axial planes add more accuracy for the meniscal tear diagnosis with $79 \%$ accuracy for the medial meniscal tears and $71 \%$ for the lateral meniscus tears [6].

In another study done by Ohishi et al., he used 3D data and reconstructed axial images and found they are helpful in the diagnosis of tears. His study showed a high false positive rate in the diagnosis of medial meniscal tears and could not detect horizontal tears [10].

Gokalp et al. made a similar study comparing the sagittal, coronal, and axial images to arthroscopy. For

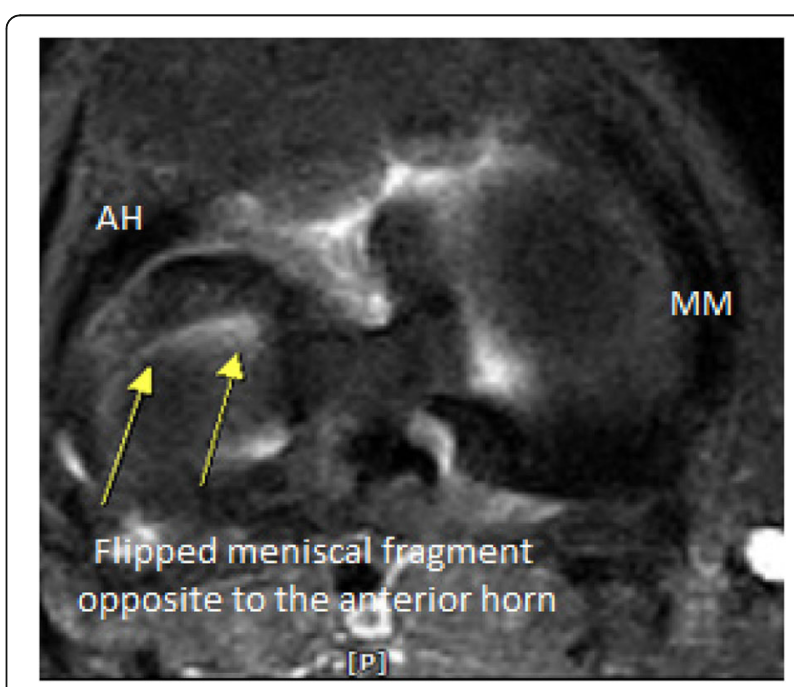

Fig. 8 Displaced bucket handle tear of the lateral meniscus (arrows). Note ventrally flipped meniscal fragment within the lateral femorotibial joint compartment mildly compressing the free meniscal edge of the anterior horn. $\mathrm{AH}=$ anterior horn and $\mathrm{MM}=$ medial meniscus 


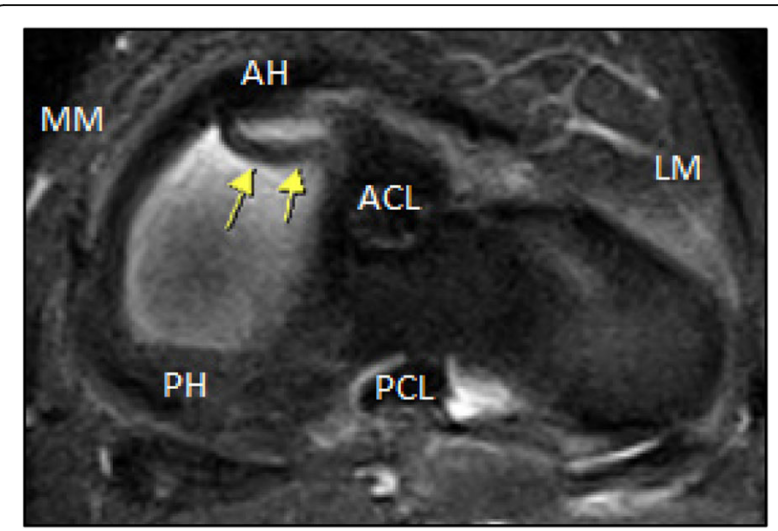

Fig. 9 Axial fat PD WI revealed a displaced flap tear. Note the rather attenuated body secondary to displaced meniscal flap tear from the free meniscal edge, in the anterior aspect of the medial femorotibial joint compartment. $\mathrm{LM}=$ lateral meniscus, $\mathrm{MM}=$ medial meniscus, $\mathrm{AH}=$ anterior horn, $\mathrm{PH}=$ posterior horn, $\mathrm{PCL}=$ posterior cruciate ligament, and $\mathrm{ACL}=$ anterior cruciate ligament

sagittal PD WIs, the sensitivity was $90.62 \%$ and specificity $70.73 \%$ for the medial meniscal tears while for the lateral meniscus the sensitivity was $72.73 \%$ and specificity $77.1 \%$. For axial images, the sensitivity was $97.3 \%$ for the medial meniscal tears and $84 \%$ specificity while for the lateral meniscal tears sensitivity was $95.65 \%$ and specificity $80.56 \%$ [11].

In our study, we compared the findings of thin axial images alone with arthroscopic findings. Of course, each MR study included sagittal and coronal images, but they were not included in the diagnosis of tears. Experienced musculoskeletal radiologists can diagnose all types of tears using axial images with more accurate diagnosis by using the sagittal and coronal cuts as well.

Axial PD WIs yield a high diagnostic performance with sensitivity of $96.3 \%$ for the medial and lateral meniscal tears and specificity of $100 \%$. All lateral meniscal tears were accurately diagnosed by the axial images with $100 \%$ sensitivity and specificity.

A horizontal tear of the medial meniscus was missed in this study [out of three] because it was a small and thin tear running parallel to the axial plane taken in our study. Two other horizontal tears in medial meniscus were diagnosed by the axial images as they were large and thick dissecting the horizontal plane and so they readily appeared in the axial cuts. No horizontal tears encountered in the lateral meniscus.

The morphology of the different types of tear is very important in the management and not only in the classifications. Orientation of the lesion, gapping, direction, and different extensions are crucial in the decision making of either suturing the tear or doing meniscectomy, and this can only be assessed by the axial images.
One limitation of the study is that the time of scan was 6-8 min which was considered a relatively long duration, but it yields high diagnostic capability that weighs a long time. Using high field 3-T systems can reduce the acquisition time. Another limitation was that we did not use the sagittal and coronal images in the diagnosis which could give more accurate results with arthroscopy findings, but we wanted to emphasize the importance of the axial images.

In conclusion, axial PD WIs yield high diagnostic capability and give valuable information about the types of tears, and it is worth to add this sequence as a routine in knee MRI imaging together with the other conventional sequences but not to replace them.

\section{Abbreviations \\ FS PD: Fat saturated proton density; GRASS: Gradient-recalled acquisition in the steady state; MR: Magnetic resonance; PCL: Posterior cruciate ligament; Wls: Weighted images}

\section{Acknowledgements}

Not applicable

\section{Authors' contributions}

YI participated in the sequence alignment, drafted the manuscript, participated in the design of the study, and performed the statistical analysis. MA and MN participated in collecting the data and images and in the manuscript revision. All authors read and approved the final manuscript.

\section{Funding}

No funding sources.

Availability of data and materials

The datasets used and/or analyzed during the current study are available from the corresponding author on reasonable request.

Ethics approval and consent to participate

The study was approved by the institutional ethics committee and all required consents were taken.

\section{Consent for publication}

Required consents were taken.

\section{Competing interests}

The authors declare that they have no competing interests.

\section{Author details}

${ }^{1}$ Radiodiagnosis Department, Ain Shams University, 3/4 Borg Elhamd Anwer Elmofty Street, Nasr City, Cairo, Egypt. ${ }^{2}$ Radiodiagnosis Department, Helwan University, Cairo, Egypt. ${ }^{3}$ Radiodiagnosis Department, Ain Shams University, Cairo, Egypt.

Received: 13 June 2019 Accepted: 16 July 2019

Published online: 02 September 2019

\section{References}

1. Ryzewicz M, Peterson B, Siparsky PN, Bartz RL (2007) The diagnosis of meniscus tears: the role of MRI and clinical examination. Clin Orthop Relat Res 455:123-133

2. Jee WH, McCauley TR, Kim JM, Jun DJ, Lee YJ, Choi BG et al (2003) Meniscal tear configurations: categorization with MR imaging. AJR Am J Roentgenol 180:93-97

3. Magee T, Williams D (2006) 3.0-T MRI of meniscal tears. AJR Am J Roentgenol 187:371-375

4. Helms CA (2002) The meniscus: recent advances in MR imaging of the knee AJR Am J Roentgenol 179:1115-1122 
5. Jung JY, Yoon YC, Kwon JW, Ahn JH, Choe BK (2009) Diagnosis of internal derangement of the knee at 3.0-T MR imaging: 3D isotropic intermediateweighted versus 2D sequences. Radiology 253:780-787

6. Tarhan NC, Chung CB, Mohana-Borges AV, Hughes T, Resnick D (2004) Meniscal tears: role of axial MRI alone and in combination with other imaging planes. AJR Am J Roentgenol 183:9-15

7. Yoon YC, Kim SS, Chung HW, Choe BK, Ahn JH (2007) Diagnostic efficacy in knee MRI comparing conventional technique and multiplanar reconstruction with one-millimeter FSE PDW images. Acta Radiol. 48:869-874

8. Fox MG (2007) MR imaging of the meniscus: review, current trends, and clinical implications. Radiol Clin North Am 45:1033-1053

9. Araki Y, Ootani F, Tsukaguchi I, Ootani M, Furukawa T, Yamamoto T et al (1992) MR diagnosis of meniscal tears of the knee: value of axial threedimensional Fourier transformation GRASS images. AJR Am J Roentgenol 158:587-590

10. Ohishi T, Takahashi M, Abe M, Tsuchikawa T, Mori M, Nagano A (2005) The use of axial reconstructed images from three-dimensional MRI datasets for morphologic al diagnosis of meniscal tears of the knee. Arch Orthop Trauma Surg 125:622-627

11. Gökalp G, Nas O, Demirag B, Yazici Z, Savci G (2012) Contribution of thinslice $(1 \mathrm{~mm})$ axial proton density MR images for identification and classification of meniscal tears: Correlative study with arthroscopy. Br J Radiol 85:e871-e878. https://doi.org/10.1259/bj/74041910

\section{Publisher's Note}

Springer Nature remains neutral with regard to jurisdictional claims in published maps and institutional affiliations.

\section{Submit your manuscript to a SpringerOpen ${ }^{\circ}$ journal and benefit from:}

- Convenient online submission

- Rigorous peer review

- Open access: articles freely available online

- High visibility within the field

- Retaining the copyright to your article

Submit your next manuscript at $\boldsymbol{\nabla}$ springeropen.com 